\title{
PREVALÊNCIA DA INFECÇÃO PELO PAPILOMAVÍRUS HUMANO (HPV) EM MULHERES DO SUDESTE DO ESTADO DO PARÁ
}

\section{Sheyla Elisa Castro Negrão}

Fisioterapeuta. Graduada pela Universidade da Amazônia (UNAMA), Belém (PA), Brasil.

\section{Wirlene Cardoso Dias}

Fisioterapeuta. Graduada pela Universidade da Amazônia (UNAMA), Belém (PA), Brasil.

\section{Dayse Danielle de Oliveira Silva}

Fisioterapeuta. Doutora em Ciências da Reabilitação pela Universidade Nove de Julho, Docente do Departamento de Ciências do Movimento Humano da Universidade do Estado do Pará, Belém (PA), Brasil.

\section{Erica Feio Carneiro Nunes}

Fisioterapeuta. Doutora em Ciências da Reabilitação pela Universidade Nove de Julho, Docente do Departamento de Ciências do Movimento Humano da Universidade do Estado do Pará, Belém (PA), Brasil.

\section{Ana Júlia Cunha Brito}

Fisioterapeuta. Mestre em Desenvolvimento e Meio Ambiente Urbano pela Universidade da Amazônia, Docente da Faculdade Cosmopolita, Belém (PA), Brasil.

\section{Biatriz Araújo Cardoso}

Fisioterapeuta, Doutora em Ciências pelo Programa de Medicina Tropical pelo Instituto Oswaldo Cruz FIOCRUZ, Docente do Departamento de Ciências do Movimento Humano da Universidade do Estado do Pará, Belém (PA), Brasil.

\section{George Alberto da Silva Dias}

Fisioterapeuta, Doutor em Doenças Tropicais pelo NMT/UFPA/PA, Docente do Departamento de Ciências do Movimento Humano da Universidade do Estado do Pará, Belém (PA), Brasil.
RESUMO: Investigar a prevalência da infecção pelo Papilomavirus bumano (HPV) em mulheres do município de Jacundá (PA) e fatores de risco para esta infecção. Estudo observacional, descritivo e analítico, do tipo transversal com coleta em prontuários de mulheres atendidas no Hospital Municipal e consultório particular de ginecologia, oriundos de Jacundá entre 2015 e 2016. Aplicou-se análise descritiva pelo programa Epi Info 3.5.1 e BioEstat 5.0. Dos 235 prontuários, 36,6\% possuíam HPV e aquelas com 45 anos ou mais possuíam maior prevalência desta infecção (40,3\%). Quanto à citologia, $28,9 \%$ possuíam alguma alteração. E somente as variáveis escolaridade, etilismo e citologia alterada possuíam associação significante, exclusivamente para as de 26 a 44 anos, sendo variáveis consideradas como fatores de risco para aquisição e manutenção viral. Assim, houve alta prevalência de HPV na região ( 45 anos ou mais) com fatores de risco relacionados à escolaridade, ao etilismo e às alterações na citologia cervical, na faixa etária de 26 a 44 anos.

PALAVRAS-CHAVE: Doenças sexualmente transmissíveis; Epidemiologia; Displasia do colo do útero.

\section{PREVALENCE OF HUMAN PAPILLOMAVIRUS INFECTION (HPV) IN FEMALES FROM THE SOUTHEASTERN RE- GION OF THE STATE OF PARÁ, BRAZIL}

ABSTRACT: The prevalence of infection by human papillomavirus (HPV) in females of Jacundá PA Brazil and its risk factors are discussed. Current observational, descriptive, analytic and transversal study of clinical charts of females from the municipality of Jacundá and attended to in the Municipal Hospital and private clinic was undertaken between 2015 and 2016. Descriptive analysis was applied by programs Epi Info 3.5.1 and BioEstat 5.0. Further, $36.6 \%$ of people in 235 charts had HPV; females aged 45 years or more had the greatest prevalence (40.3\%) of the infection; $28.9 \%$ had alterations in cytology. Variables such as schooling, alcoholism and altered cytology had significant association, exclusively for people between 26 and 44 years old. They are variables considered to be risk factors for the acquisition and maintenance of the virus. High prevalence of HPV occurred in the region (with 45 years old and older) with risk factors related to schooling, alcoholism and cervix cytology changes in the 26-44 years age bracket.

KEY WORDS: Sexually transmitted diseases; Epidemiology; Uterine cervical dysplasia. 


\section{INTRODUÇão}

Infecções genitais por determinados tipos de Papilomavirus humano (HPV) estão envolvidas no desenvolvimento de lesões cervicais. Apesar, de serem conhecidas desde a antiguidade, estas infecções virais começaram a chamar atenção a partir da década de 1980 , quando se correlacionou o câncer do colo de útero às lesões causadas pela infecção por HPV ${ }^{1}$.

É um vírus de transmissão sexual e sua prevalência vem aumentando consideravelmente em todo o mundo decorrente de inúmeros fatores. Por se tratar de uma doença sexualmente transmissível (DST) é compreensível que a iniciação sexual precoce, a multiplicidade de parceiros sexuais e o sexo desprotegido estejam entre os principais contribuintes para tal crescimento. A maioria das mulheres sexualmente ativas terá contato com o vírus durante algum momento da vida. Entretanto, mais de 90\% dessas novas infecções regridem espontaneamente em seis a 18 meses, muitas delas desencadeadas pela própria resposta imunológica do hospedeiro ${ }^{2,3}$.

A transmissão viral se dá pelo contato íntimo e com os fluídos durante o ato sexual e, uma vez infectada, a mulher pode permanecer com o vírus latente durante anos no organismo, dificultando assim a descoberta precoce para realização de um tratamento efetivo. O epitélio do colo do útero de uma mulher jovem em relação ao de uma adulta, está mais propenso a infecções pela falta de maturação das células metaplásicas, sofrendo assim alterações significativas de acordo com a quantidade de agressões que vier a sofrer causadas pelo vírus. Infecções persistentes por HPV podem levar a transformações intraepiteliais progressivas evoluindo para lesões precursoras do câncer, as quais, se não diagnosticadas e tratadas oportunamente, evoluem para o câncer do colo do útero propriamente dito. Além disso, outros fatores estão implicados no desenvolvimento desta doença quando associada à infecção por HPV como, por exemplo, o tabagismo e a imunodepressão causada pelo Vírus da Imunodeficiência Humana (HIV) ${ }^{3,4}$.

O HPV possui mais de 120 genótipos identificados e classificados em oncogênicos e não oncogênicos, entre estes 40 têm afinidade pela região anugenital. Existem, atualmente, 13 tipos virais reconhecidos como oncogênicos e desses, os mais comuns são o HPV16 e o HPV18. Estima-se que 600 milhões de pessoas tenham sido infectadas no mundo todo e no Brasil estas estimativas variam entre $13,7 \%$ a $54,3 \%$ mulheres, as de 15 e 25 anos são as mais acometidas, o que faz do Brasil um dos líderes mundiais em incidência para esta infecção $0^{5-7}$.

As estimativas do Instituto Nacional de Câncer (INCA) indicam que no ano de 2016, no Brasil, foram esperados 16.340 novos casos de câncer de colo do útero com um risco estimado de 15,85 casos a cada 100 mil mulheres, sendo o mais incidente na região Norte (23,97/100 mil). O Pará tem uma taxa estimada de 20,52 casos para cada 100 mil mulheres ${ }^{3}$.

Desde a década de 1970, em países desenvolvidos, os programas de prevenção na saúde da mulher por meio de exames de colpocitologia oncótica ou Papanicolau, realizados regularmente e com tratamento adequado das lesões pré-cancerosas, são eficazes na redução da incidência de doenças e sua mortalidade. Os mesmos são capazes de reduzir em cerca de $80 \%$ sua incidência com um programa organizado de rastreamento, entretanto, esta abordagem é de alto custo e pode ser difícil de implementar e manter em locais de recursos escassos. Visando o combate da disseminação do vírus e o controle das lesões induzidas pelo HPV, foram desenvolvidos dois tipos de vacinas, a profilática e a terapêutica, porém esta última ainda se mostra com baixa eficácia ${ }^{8,9}$.

Atualmente, a saúde da mulher é prioridade, no entanto deve-se considerar a heterogeneidade que caracteriza o Brasil, pois há disparidade nas questões socioeconômicas e culturais, bem como relacionadas ao acesso às ações e aos serviços de saúde. Diante disso, existe diferença considerável no perfil epidemiológico da população feminina de uma região à outra do país. Estudos que envolvam estas questões podem fornecer subsídios para se traçar estratégias voltadas para fortalecer a atuação das políticas públicas de saúde, principalmente quando se trata do processo saúde-doença da população do interior do Estado ${ }^{10}$. Assim, o objetivo deste estudo foi investigar a prevalência da infecção pelo Papilomavirus bumano (HPV) em mulheres do município de Jacundá e fatores de risco para esta infecção. Estes dados podem fornecer valiosas informações acerca desta infecção viral no interior do Estado do Pará onde programas regionais relacionados à prevenção e manejo desta infecção ainda são escassos. 


\section{METODOLOGIA}

\section{ASPECTOS ÉTICOS}

A pesquisa teve início após aprovação pelo Comitê de Ética em Pesquisa da Universidade da Amazônia (Unama) (parecer $\mathrm{n}^{\mathrm{o}}$ 1.946.234). Trata-se de um estudo observacional, descritivo, do tipo transversal seguindo as normas da resolução 466/12 do Conselho Nacional de Saúde, relativa à pesquisa com seres humanos. Os dados foram coletados de prontuários de mulheres que deram entrada no serviço de ginecologia e obstetrícia, no Hospital Municipal de Jacundá e, também, em consultório particular, ambos do município de Jacundá (PA), sendo selecionados por meio da amostragem não probabilística por conveniência. Todos os prontuários analisados tiveram anuência da médica responsável e dos pesquisadores por meio da assinatura do Termo de Compromisso de Utilização de Dados (TCUD).

\section{AMOSTRA}

Foram analisados 235 prontuários de mulheres entre os anos de 2015 e 2016, sendo incluídos no estudo todos os prontuários com o resultado de colposcopia e exame de Papanicolau para verificar a infecção por HPV, maiores de 18 anos, com história de vida sexual iniciada e excluídos prontuários incompletos e sem identificação médica responsável pelos prontuários.

\section{PROCEDIMENTOS}

Os dados foram coletados em formulário-padrão criado pelos pesquisadores, incluindo as seguintes variáveis: nome, idade, profissão, estado civil, procedência, grau de lesão, quantidade de parto, número de abortos, diagnóstico (CID), presença ou ausência da infecção por HPV e morbidades associadas ao vírus.

No Hospital Municipal de Jacundá, as informações colhidas deram-se em ambiente reservado estando presente apenas os pesquisadores e a médica responsável pelos dados das pacientes, que os auxiliou no manejo e avaliação das informações clínicas quando não identificadas de maneira correta. Na coleta de dados no con- sultório particular, foram tomados os mesmos cuidados realizados no hospital.

\section{ANÁLISE DOS DADOS}

Para análise estatística das características clínica e epidemiológica, as variáveis categóricas foram apresentadas como frequências, e as numéricas, por meio de medidas de tendência central e de dispersão. Na investigação mais detalhada dos fatores de risco possivelmente associados à infecção por HPV, realizou-se análise geral e estratificada por idade, assim procedeu-se análise por três faixas etárias ( 18 a 25 anos, 26 a 44 anos e 45 ou mais anos), sendo então empregado, na análise bivariada, o cálculo das razões de chances de prevalência (ORp) com intervalo de confiança (IC) de 95\%, sendo a significância estatística verificada pelo teste do Qui-quadrado (2) e/ou exato de Fisher com um nível alfa $5 \%(\mathrm{p} \leq 0,05)$. Todo o processamento estatístico foi realizado nos softwares Epi Info 3.5.1 e BioEstat 5.0.

\section{RESULTADOS}

Foram selecionados inicialmente 491 prontuários, entretanto 256 foram excluídos de acordo com os critérios de inclusão e exclusão da pesquisa. Assim, a análise final incluiu 235 prontuários com média de idade de $38,4 \pm 11,1$ anos e mediana de 38 anos. Quando estratificado por faixa etária, 31 mulheres estavam entre 18 a 25 anos (média: 22,3 \pm 2,1 anos; min: 18 anos; máx: 25 anos; amplitude: 7,0), 132 na faixa etária de 26 a 44 anos (média: 35,0 \pm 5,5 anos; min: 26 anos; máx: 44 anos; amplitude: 18,0) e 72 na faixa etária de 45 ou mais (média: 51,5 \pm 6,5 anos; min: 45 anos; máx: 76 anos; amplitude: $31,0)$, em que a maioria das mulheres está em situação conjugal estável, com ensino fundamental completo ou superior e trabalhando como donas de casa. Com relação à história clínica, a grande maioria dos prontuários apresentou mulheres com até duas gestações, sem história de DST, porém com presença de sintomas genitais e citologia normal e/ou inflamatória, quanto aos hábitos de vida o etilismo e tabagismo foram negados (Tabela 1).

Entre os 235 prontuários estudados, realizou-se estratificação em grupos etários. Para os três grupos, a situação conjugal estável foi mais prevalente, foram encon- 
tradas até duas gestações e sem história de DST. Na citologia, a maioria apresentou-se normal e/ou inflamatório e nos fatores comportamentais como etilismo e tabagismo, a maioria não bebia ou fumava. Com relação à escolaridade, somente no grupo de 45 anos ou mais, o ensino fundamental ou inferior ao mesmo foi mais evidente. Outro dado relevante foi em relação aos sintomas genitais, em todas as faixas etárias houve alta prevalência, porém somente entre as mulheres de 18 a 25 anos, estes sintomas estavam presentes em 100\% dos prontuários (Tabela 2).

De forma geral 36,6\% (86/235) dos prontuários analisados apresentavam diagnóstico de infecção genital pelo HPV. Quando estratificou, percebeu-se que 38,7\% (12/31) estavam presentes entre as mulheres de 18 a 25 anos, 34,1\% (45/132) entre as de 26 a 44 anos e 40,3\% (29/72) entre as mulheres acima de 45 anos (Tabela 3).

Em relação à citologia cervical, no geral, $71,1 \%$ (167/235) apresentaram-se normal/inflamatório e 28,9\% (68/235) com alterações (Tabela 1). Quando se pesquisou a presença viral nos resultados da citologia cervical, nas mulheres sem lesão o HPV estava presente em 50\% (1/2) delas, naquelas com NIC I 89,3\% (25/28), com NIC II e III 93,3\% (14/15), e nas diagnosticadas com carcinoma o HPV estava presente em 100\% (3/3), conforme ilustrado na Tabela 4.

A Tabela 5 mostra a prevalência entre a presença de HPV e os fatores de risco sociodemográficos considerando as faixas etárias. Com relação à situação conjugal, não foi observado relação estatisticamente significante. Porém, quando analisada a escolaridade este fator se mostrou associado à infecção viral, sendo que somente as mulheres de 26 a 44 anos que possuíam menos de nove anos de escolaridade tinham chance de 2,69 (IC95\%: 1,28 $-5,66 ; \mathrm{p}=0,01$ ) vezes maior de estar infectadas pelo HPV do que aquelas que apresentaram escolaridade superior a nove anos.

Em relação aos fatores comportamentais, ginecológicos e reprodutivos, somente o fator etilismo se mostrou associado à infecção pelo HPV no grupo de 26 a 44 anos, em que as mulheres que bebiam tinham chance de 3,8 (IC95\%: 1,77 - 8,26; $\mathrm{p}<0,001$ ) vezes maior de estar infectadas pelo HPV do que aquelas que não bebiam álcool (Tabela 5).

Por fim, houve relação significante da infecção por HPV e as alterações citológicas. De acordo com a faixa etária, somente entre as mulheres de 26 a 44 anos com citologia alterada possuíam chance de 10,8 (IC95\%: $4,37-26,82 ; \mathrm{p}<0,001)$ vezes maior de estar infectadas pelo HPV do que aquelas que se apresentaram normal ou inflamatória (Tabela 5).

\section{DISCUSSÃO}

A prevalência de HPV entre os prontuários analisados mostrou-se elevada e quando estratificado por faixa etária, a idade mais prevalente ficou entre as mulheres de 45 anos ou mais, seguida entre aquelas de 18 a 25 anos. Este dado é importante principalmente quando se trata do risco para o desenvolvimento de lesões cervicais, muito intimamente relacionadas ao sistema imunológico. Os dois extremos vistos neste estudo nos fazem elucubrar que mulheres com idades avançadas e aquelas que são jovens estão com o sistema imunológico ou em processo de declínio ou em construção, estando suscetíveis ao grande risco de infecção e ao desenvolvimento das lesões associadas ao HPV.

No Brasil, estima-se que o câncer de colo uterino seja o terceiro mais comum na população feminina. Representa a segunda causa de óbito entre mulheres, no ano de $2002^{11}$. O Estado do Pará apresenta uma das maiores prevalências de câncer de colo uterino no Brasil ${ }^{12}$, assim estudos que busquem informações sobre agentes infecciosos precursores do câncer são de grande importância, principalmente, quando se trata de populações do interior do Estado.

A detecção precoce da infecção pelo HPV é essencial para que medidas terapêuticas mais eficazes sejam realizadas a fim de promover o controle viral e prevenção de lesões cervicais, principalmente quando se trata de mulheres jovens. Segundo um estudo ${ }^{13}$ a prevalência da infecção genital por HPV foi mais evidente em mulheres de 15 a 25 anos, isso pode ser justificado pelo início precoce da vida sexual e estar mais exposta ao vírus. Porém, este dado difere do que foi observado neste estudo, onde houve predominância na faixa etária de 45 ou mais, porém a segunda maior prevalência foi em mulheres de 18 a 25 anos, fato este que pode levar a considerar que a iniciação sexual precoce é um fator predisponente para esta infecção na população estudada. 
Com relação à situação conjugal, a maioria tinha união estável permitindo a dedução da existência de maior atividade sexual monogâmica e fixa, supondo que a exposição ao vírus foi menor durante sua vida, ou seja, quanto maior a estabilidade conjugal menor é a chance de se infectar pelo HPV e este achado pode ser sustentado neste estudo pela inexistência da associação entre HPV e o estado civil. No entanto, nem sempre a união estável é fator protetor para se contrair o vírus. Um estudo ${ }^{14}$ mostrou que a prevalência de HPV em mulheres casadas ou em união estável era alta, e apontam este fato por confiarem em seus parceiros deixando de usar preservativos, permitindo concluir que nem sempre a estabilidade na relação seja sinônimo de fidelidade.

Ter um bom nível de entendimento é essencial para prevenção das DSTs. Ao se analisar esta variável, foi possível verificar relação direta entre escolaridade e o risco de infecção viral. Houve associação significante somente na faixa etária entre 26 a 44 anos com menos de nove anos de escolaridade, com chance de 2,69 vezes mais de estar infectadas pelo HPV, ou seja, mulheres com baixa escolaridade nesta faixa etária é uma população com maior risco de se infectar pelo vírus do que aquelas com escolaridade alta. Este resultado corrobora com outro estudo ${ }^{15}$ onde encontraram maior prevalência por HPV nas mulheres com menor escolaridade, permitindo considerar que menor instrução ocasiona déficit de conhecimento quanto à prevenção relacionada às DSTs.

Outros tipos de DSTs também são fatores de risco para infecção do HPV, até mesmo porque a via de contágio é a mesma ${ }^{16} \mathrm{e}$ alguns sintomas genitais são característicos destas doenças. Na maioria dos prontuários analisados, em todas as faixas etárias, as mulheres apresentavam algum tipo de sintoma genital, porém nem todas relatavam história de DST. Diante deste fato, no presente estudo, não foi possível observar associação entre estas variáveis e o risco de infecção viral.

Sabe-se também que hábitos de vida saudáveis são essenciais para uma boa reposta imunológica do hospedeiro. O tabagismo é um fator de risco associado tanto para a infecção genital pelo HPV como para o desenvolvimento de câncer de colo uterino ${ }^{17,18}$. Muitos prontuários mostravam que a maioria das mulheres não fumava, e, em menor intensidade, também não ingeriam bebidas alcoó- licas. No presente estudo foi observado que não houve associação entre o tabagismo e o risco de infecção viral, o que pode ser uma característica das mulheres dessa região o não uso do cigarro. Porém, mesmo a maioria das mulheres relatando que não bebiam, observou-se relação entre o etilismo e a chance de infecção viral. Dentre as faixas etárias estudadas, somente as do grupo de 26 a 44 anos mostraram chance de 3,8 vezes maior de estar infectadas pelo HPV do que aquelas que não ingeriam álcool. Este achado corrobora com outro estudo ${ }^{18}$ onde relata que o problema está nas mulheres que estão com o sistema imunológico comprometido pelo excesso de álcool predispondo a infecção viral.

O número de gestações pode ser outra variável que leva ao risco de infecção pelo HPV, pois se faz deduzir que houve exposição da mucosa vaginal ao órgão genital do homem infectado. No presente estudo, a maioria das mulheres relatou ter até duas gestações, e não houve nenhuma relação significativa com a infecção por HPV. Dados do IBGE $^{19}$, para todo o Brasil, apontam taxa de fecundidade geral de 2,4 filhos por mulher, sendo que, no Estado do Pará, na zona rural encontraram-se índices superiores, em torno de 4,6 filhos. No entanto, este dado foi o contrário do que se esperava, e, talvez por isso, não foi possível chegar a associação para esta variável.

Em relação à citologia cervical, houve predomínio de diagnóstico de normalidade e/ou inflamatório. No entanto, quando se analisou a citologia alterada nas três faixas etárias com a chance de estarem infectados pelo vírus, somente entre as mulheres de 26 a 44 anos tinham chance de 10,8 vezes maior de estar infectadas pelo HPV. Muitas mulheres desconhecem ou não realizam o exame Papanicolau regularmente pela situação socioeconômica em que se encontram ${ }^{20}$. No entanto, é essencial este exame para o acompanhamento da saúde da mulher bem como intervenções precoces, visto que neste estudo dentre as citologias caracterizadas como inflamatórias 23\% tinham HPV, das consideradas com NIC I 89,3\% estavam infectadas pelo vírus, NIC II ou III 93,3\% apresentavam HPV e aqueles prontuários diagnosticados com carcinoma $100 \%$ o HPV estava presente.

Este estudo consistiu em análise de dados secundários coletados em 235 prontuários de mulheres atendidas em hospital e consultório particular de ginecologia, 
ambos situados no município de Jacundá a sudeste do Estado do Pará. Inicialmente deveriam também ser rastreados prontuários de anos anteriores a 2015, no entanto, foi observado que muitos estavam acondicionados em condições adversas ou não foram encontrados registros destes anos. Muitos dados também foram perdidos pela falta de manejo criterioso para o arquivamento dos mesmos ou pelo preenchimento incompleto levando à exclusão da grande parte dos prontuários coletados $(n=256)$, levantando a hipótese de que os dados referentes à infecção por HPV nesta região estão subestimados. Há a necessidade de se mostrar a importância de se preservar estes dados, bem como seu preenchimento completo, pois poderão servir de base para ações em saúde direcionadas a este público específico.

As limitações deste estudo estão relacionadas, sobretudo, no preenchimento e armazenamento dos prontuários para a coleta de dados referentes aos fatores de risco para aquisição e manutenção do HPV. As análises estatísticas que mostram associação do vírus com fatores de risco, foram realizadas exclusivamente com dados coletados dos prontuários relativos às informações dadas pelas mulheres com possível ocultação da verdade e/ou mascaramento de resposta.

\section{CONCLUSÃO}

Este estudo possibilitou a avaliação de um número significativo de prontuários de mulheres oriundas do município de Jacundá. De acordo com registros encontrados houve alta prevalência de mulheres infectadas pelo HPV na região, e quando se estratificou por faixa etária, observou-se maior destaque para aquelas de 45 anos ou mais seguido da faixa etária de 18 a 25 anos. No entanto, os fatores de risco, para o incremento da vulnerabilidade feminina quanto à infecção viral, estavam voltados para escolaridade, etilismo e alterações na citologia cervical, exclusivamente para a faixa etária de 26 a 44 anos.

Os dados encontrados permitem refletir sobre o HPV ao longo da história, tornando-se um problema de saúde pública. Acredita-se que estudos desta natureza podem fornecer informações para elaboração de estratégias regionalizadas que visem a promoção à saúde da mulher, educação sexual de jovens e adolescentes, e prevenção de DST.

\section{REFERÊNCIAS}

1. Tulio S, Pereira LA, Neves F, Pinto A. Relação entre a carga viral de HPV oncogênico determinada pelo método de captura hibrida e o diagnóstico citológico de lesões de alto grau. J BrasPatolMed Lab. 2007;43(1):31-5.

2. CamposAN. Aspectos clínicos e epidemiológicos da infecção genital pelo papilomavírus humano (HPV) em adolescentes da região metropolitana de Belém[dissertação]. Belém: Universidade Federal do Pará; $2010.83 \mathrm{p}$.

3. Instituto Nacional do Câncer. Coordenação de Prevenção e Vigilância. Estimativa 2016: Incidência no câncer no Brasil. Rio de Janeiro; 2016.

4. Macedo FLS, Silva ER, Soares LRC, Rosal VMS, Carvalho NAL, Rocha MGL. Infecção pelo HPV na adolescente. Femina.2015;43(4):185-88.

5. Tamayo-Acevedo LS, Gil-Cano PA, Tamayo-Acevedo, LE. Lo que no se ve, no existe: percepciones sobre cáncer y papilomavirus humano enjóvenesuniversitarios, Medellín, Colombia, 2014. Aquichan. 2015;15(2):253-70.

6. Coser J, Fontoura S, Belmonte C, Vargas VRA. Relação entre fatores de risco e lesão precursora do câncer do colo do útero em mulheres com e sem ectopia cervical. RBAC. 2012;44(1):50-4.

7. Gaspar J, Quintana SM, Reis RK, Gil E. Fatores sociodemográficos e clínicos de mulheres com papilomavírus humano e sua associação com o vírus da imunodeficiência humana. Rev. Latino-Am. Enfermagem. 2015;23(1):74-81.

8. Giraldo PC, Silva MJPMA, Fedrizzi EN, Gonçalves AKS, Amaral RLG, Eleutério Junior J, et al. Prevenção da infecção por HPV e lesões associadas com o uso de vacinas. DST - J BrasDoenças Sex Transm. 2008;20(2):132140.

9. Zardo GP, Farah FP, Mendes FG, Franco CAGS, Molina GVM, Melo GN, et alVacina como agente de imunização contra o HPV. Ciência \& Saúde Coletiva. 2014;19(9):3799-3808.

10. Brasil. Ministério da Saúde. Centro Brasileiro de Análise e Planejamento. Pesquisa Nacional de Demografia e Saúde da Criança e da Mulher - PNDS 2006: dimensões do processo reprodutivo e da saúde da 
criança. Brasília: Ministério da Saúde; 2009.

11. Brasil. Ministério da Saúde. Secretaria de Atenção à Saúde. Instituto Nacional de Câncer. Coordenação de Prevenção e Vigilância de Câncer. Prevenção do câncer do colo do útero. Normas e recomendações do INCA. RevBrasCancerol. 2003;49:205.

12. Brasil. Ministério da Saúde. Secretaria de Atenção à Saúde. Instituto Nacional de Câncer. Estimativas 2010: incidência de câncer no Brasil. Rio de Janeiro: Instituto Nacional de Câncer; 2009.

13. Giuliano AR, Papenfuss M, Abrahamsen M, Denman C, Zapien JG, Henze JL, et al. Human papillomavirus infection at the United States-Mexico border: implications for cervical cancer prevention and control. Cancer Epidemiol Biomarkers Prev.2001;10(11):112936.

14. Silva A, Gonçalves A, Katherine S, Giraldo PC, Pontes AC, Dantas GL, et al. A eficácia da vacina profilática contra o HPV nas lesões HPV induzidas. Femina. 2009;37(10):519-26.

15. Kornya L, Cseh I, Deak J, Bak M, Fulop V. The diagnostics and prevalence of genital Human papillomavirus (HPV) infection in Hungary. Eur J ObstetGynecolReprod Biol. 2002;100(2):231-36.

16. Saha R, Thapa M. Correlation of cervical cytology with cervical histology. Kathmandu Univ Med J. 2005;3(3):222-4.

17. Nuñez J, Delgado M, Pino G, Girón H, Bolet B. Smoking as a risk factor for preinvasive and invasive cervical lesions in female sex workers in Venezuela. Int J Gynecol Obstet. 2002;79(1):57-60.

18. Winer RL, Lee S-K, Hughes JP, Adam DE, Kiviat NB, Koutsky LA. Genital human papillomavirus infection: incidence and risk factors in a cohort of female university students. Am J Epidemiol. 2003;157(3):21826.

19. Instituto Brasileiro de Geografia e Estatística - IBGE. Censo Demográfico 2000. Brasília: IBGE; 2004.

20. Trottier H, Franco EL. The epidemiology of genital human papillomavirus infection. Vaccine. 2006;30:Suppl 1:S1-15.

\section{ANEXOS - Tabelas}

Tabela 1. Distribuição das mulheres do estudo de acordo com fatores de risco selecionados, para todo o grupo $(\mathrm{N}=235)$, Jacundá - PA, 2015 a 2016

\begin{tabular}{lll}
\hline \multirow{2}{*}{ Variáveis } & \multicolumn{2}{c}{ Mulheres } \\
\cline { 2 - 3 } & $\mathrm{N}=235$ & $\%$ \\
\hline
\end{tabular}

\begin{tabular}{lcc}
\hline Situação conjugal & & \\
Solteira & 41 & $17,4 \%$ \\
Casada/União estável & 181 & $77 \%$ \\
Separada/divorciada & 10 & $4,3 \%$ \\
Viúva & 3 & $1,3 \%$ \\
\hline
\end{tabular}

\begin{tabular}{ccc}
\hline Escolaridade & & \\
\hline Analfabeta & 79 & $33,6 \%$ \\
$<9$ anos de escolaridade & 38 & $16,2 \%$ \\
$>9$ anos de escolaridade & 118 & $50,2 \%$ \\
\hline
\end{tabular}

\begin{tabular}{lcc}
\hline Ocupação & & \\
\hline Dona de casa & 214 & $91,1 \%$ \\
Recepcionista & 9 & $3,8 \%$ \\
Autonoma & 11 & $4,7 \%$ \\
Aposentada & 1 & $0,4 \%$ \\
\hline Número de gestações & & \\
\hline Até 2 & 166 & $70,7 \%$ \\
3 ou mais & 69 & $29,3 \%$ \\
\hline
\end{tabular}

\begin{tabular}{ccc}
\hline História de DST & & \\
\hline Sim & 6 & $2,6 \%$ \\
Não & 229 & $97,4 \%$
\end{tabular}

\begin{tabular}{lcc} 
Não & 229 & $97,4 \%$ \\
\hline Sintomas genitais & & \\
\hline Sim & 231 & $98,3 \%$ \\
Não & 4 & $1,7 \%$ \\
\hline Tabagismo & & \\
\hline Sim & 23 & $9,8 \%$ \\
Não & 212 & $90,2 \%$ \\
\hline Etilismo & & \\
\hline Sim & 84 & $35,7 \%$ \\
$\quad$ Não & 151 & $64,3 \%$ \\
\hline Citologia & & \\
\hline Normal/nflamatório & 167 & $71,1 \%$ \\
Alterado & 68 & $28,9 \%$ \\
\hline
\end{tabular}

(DST) Doenças sexualmente transmissíveis. 
Tabela 2. Distribuição das mulheres do estudo de acordo com as faixas etárias e fatores de risco selecionados, Jacundá (PA), 2015 a 2016

\begin{tabular}{|c|c|c|c|c|c|c|}
\hline \multirow{2}{*}{ Variáveis } & \multicolumn{2}{|c|}{18 a 25 anos } & \multicolumn{2}{|c|}{26 a 44 anos } & \multicolumn{2}{|c|}{45 anos ou mais } \\
\hline & $\mathrm{N}=31$ & $\%$ & $\mathrm{~N}=132$ & $\%$ & $\mathrm{~N}=72$ & $\%$ \\
\hline \multicolumn{7}{|l|}{ Situação conjugal } \\
\hline Solteira/Separada/Viúva & 9 & $29 \%$ & 31 & $23,5 \%$ & 14 & $19,4 \%$ \\
\hline Casada/União Estável & 22 & $71 \%$ & 101 & $76,5 \%$ & 58 & $80,6 \%$ \\
\hline \multicolumn{7}{|l|}{ Escolaridade } \\
\hline$<9$ anos de estudo & 11 & $35,5 \%$ & 61 & $46,2 \%$ & 45 & $62,5 \%$ \\
\hline$>9$ anos de estudo & 20 & $64,5 \%$ & 71 & $53,8 \%$ & 27 & $37,5 \%$ \\
\hline \multicolumn{7}{|l|}{ Número de gestações } \\
\hline Até 2 & 31 & $100 \%$ & 96 & $72,7 \%$ & 39 & $54,2 \%$ \\
\hline 3 ou mais & - & - & 36 & $27,3 \%$ & 33 & $45,8 \%$ \\
\hline \multicolumn{7}{|l|}{ História de DST } \\
\hline Sim & - & - & 4 & $3 \%$ & 2 & $2,8 \%$ \\
\hline Não & 31 & $100 \%$ & 128 & $97 \%$ & 70 & $97,2 \%$ \\
\hline \multicolumn{7}{|l|}{ Sintomas genitais } \\
\hline Sim & 31 & $100 \%$ & 129 & $97,7 \%$ & 71 & $98,6 \%$ \\
\hline Não & - & - & 3 & $2,3 \%$ & 1 & $1,4 \%$ \\
\hline \multicolumn{7}{|l|}{ Tabagismo } \\
\hline Sim & 2 & $6,5 \%$ & 14 & $10,4 \%$ & 7 & $9,7 \%$ \\
\hline Não & 29 & $93,5 \%$ & 118 & $89,4 \%$ & 65 & $90,3 \%$ \\
\hline \multicolumn{7}{|l|}{ Etilismo } \\
\hline Sim & 13 & $41,9 \%$ & 44 & $33,3 \%$ & 27 & $37,5 \%$ \\
\hline Não & 18 & $58,1 \%$ & 88 & $66,7 \%$ & 45 & $62,5 \%$ \\
\hline \multicolumn{7}{|l|}{ Citologia } \\
\hline Normal/Inflamatório & 22 & $71 \%$ & 98 & $74,2 \%$ & 47 & $65,3 \%$ \\
\hline Alterado & 9 & $29 \%$ & 34 & $25,8 \%$ & 25 & $34,7 \%$ \\
\hline
\end{tabular}

(-) Dados numéricos igual a zero.

(DST) Doenças sexualmente transmissíveis

Tabela 3. Prevalência da infecção genital por HPV na população geral e segundo as diferentes faixas etárias $(\mathrm{N}=235)$, Jacundá (PA), 2015 a 2016

\begin{tabular}{|c|c|c|c|c|c|c|}
\hline \multirow{2}{*}{ HPV } & \multicolumn{6}{|c|}{ Mulheres } \\
\hline & \multicolumn{3}{|c|}{$N=135$} & \multicolumn{3}{|c|}{$\%$} \\
\hline SIM & \multicolumn{3}{|c|}{86} & \multicolumn{3}{|c|}{$36,6 \%$} \\
\hline \multirow[t]{3}{*}{ NÃO } & \multicolumn{3}{|c|}{149} & \multicolumn{3}{|c|}{$63,4 \%$} \\
\hline & \multicolumn{2}{|c|}{$18-25$ anos $(n=31)$} & \multicolumn{2}{|c|}{26 a 44 anos $(n=132)$} & \multicolumn{2}{|c|}{45 anos a mais $(n=72)$} \\
\hline & $\mathrm{n}$ & $\%$ & $\mathrm{n}$ & $\%$ & $\mathrm{n}$ & $\%$ \\
\hline SIM & 12 & $38,7 \%$ & 45 & $34,1 \%$ & 29 & $40,3 \%$ \\
\hline NÃO & 19 & $61,3 \%$ & 87 & $65,9 \%$ & 43 & $59,7 \%$ \\
\hline
\end{tabular}


Tabela 4. Comparação entre os resultados da citologia cervical e a prevalência do HPV para todo o grupo (N=235), Jacundá (PA), 2015-2016

\begin{tabular}{lccc}
\hline & \multirow{2}{*}{ Citologia cervical } & \multicolumn{3}{c}{ Mulheres } \\
\cline { 2 - 4 } & & $\mathbf{n}$ & HPV \\
\hline Sem lesão & 2 & 1 & $50 \%$ \\
Alterações inflamatórias & 165 & 38 & $23 \%$ \\
ASCUS & 20 & 4 & $16 \%$ \\
NIC I & 28 & 25 & $89,3 \%$ \\
NIC II e III & 15 & 14 & $93,3 \%$ \\
AGUS & 2 & 1 & $50 \%$ \\
In situ & 0 & 0 & - \\
Carcinomas & 3 & 3 & $100 \%$ \\
Outros tipos histológicos raros & 0 & 0 & - \\
\hline
\end{tabular}

(-) Dados numérico igual a zero. (ASCUS) Células Escamosas Atípicas de Significado Indeterminado. (NIC) Neoplasia Intra-Epitelial Cervical. (AGUS) Células Glandulares Atípicas de Significado Indeterminado.

Tabela 5. Prevalência da infecção genital por HPV de acordo com fatores de risco sociodemograficos, comportamento de risco, hábitos de vida e citologia cervical para a aquisição e manutenção viral, segundo as diferentes faixas etárias, Jacundá (PA) - 20152016

(Continua)

\begin{tabular}{|c|c|c|c|c|c|c|c|c|c|}
\hline \multirow{2}{*}{ Variáveis } & \multicolumn{3}{|c|}{18 a 25 anos $(n=31)$} & \multicolumn{3}{|c|}{26 a $44 \operatorname{anos}(n=132)$} & \multicolumn{3}{|c|}{45 anos ou mais $(n=72)$} \\
\hline & $\mathbf{N}$ & HPV & $\%$ & $\mathbf{n}$ & HPV & $\%$ & n & HPV & $\%$ \\
\hline \multicolumn{10}{|l|}{ Situação conjugal } \\
\hline Solteira/Separada/Viúva & 9 & 4 & $44,4 \%$ & 31 & 7 & $22,5 \%$ & 14 & 8 & $57,1 \%$ \\
\hline Casada/União Estável & 22 & 8 & $36,4 \%$ & 101 & 38 & $37,6 \%$ & 58 & 21 & $36,2 \%$ \\
\hline $\mathrm{ORp}^{1}(\mathrm{IC} 95 \%)^{2}$ & \multicolumn{3}{|c|}{$1,4(0,28-6,76)$} & \multicolumn{3}{|c|}{$0,46(0,18-1,17)$} & \multicolumn{3}{|c|}{$2,34(0,71-7,69)$} \\
\hline p-valor ${ }^{3}$ & \multicolumn{3}{|c|}{0,7039} & \multicolumn{3}{|c|}{0,1337} & \multicolumn{3}{|c|}{0,2247} \\
\hline \multicolumn{10}{|l|}{ Escolaridade } \\
\hline$<9$ anos de estudo & 11 & 4 & $36,3 \%$ & 61 & 28 & $45,9 \%$ & 45 & 17 & $37,7 \%$ \\
\hline$>9$ anos de estudo & 20 & 8 & $40 \%$ & 71 & 17 & $23,9 \%$ & 27 & 12 & $44,4 \%$ \\
\hline $\mathrm{ORp}^{1}(\mathrm{IC} 95 \%)^{2}$ & \multicolumn{3}{|c|}{$0,85(0,18-3,91)$} & \multicolumn{3}{|c|}{$2,69(1,28-5,66)$} & \multicolumn{3}{|c|}{$0,75(0,28-2,00)$} \\
\hline $\mathrm{p}$-valor ${ }^{3}$ & \multicolumn{3}{|c|}{1,0000} & \multicolumn{3}{|c|}{$0,0100^{*}$} & \multicolumn{3}{|c|}{0,6254} \\
\hline \multicolumn{10}{|l|}{ História de DST } \\
\hline Sim & 0 & 0 & 0 & 4 & 2 & $50 \%$ & 2 & 1 & $50 \%$ \\
\hline Não & 31 & 12 & $38 \%$ & 128 & 43 & $33 \%$ & 70 & 28 & $40 \%$ \\
\hline $\mathrm{ORp}^{1}(\mathrm{IC} 95 \%)^{2}$ & \multicolumn{3}{|c|}{ - } & \multicolumn{3}{|c|}{$1,97(0,26-14,51)$} & \multicolumn{3}{|c|}{$1,5(0,09-24,98)$} \\
\hline $\mathrm{p}$-valor ${ }^{3}$ & \multicolumn{3}{|c|}{1,0000} & \multicolumn{3}{|c|}{0,6052} & \multicolumn{3}{|c|}{1,0000} \\
\hline \multicolumn{10}{|l|}{ Sintomas Genitais } \\
\hline Sim & 31 & 12 & $38 \%$ & 129 & 45 & $34 \%$ & 71 & 29 & $40 \%$ \\
\hline Não & 0 & 0 & 0 & 3 & 0 & 0 & 1 & 0 & 0 \\
\hline $\mathrm{ORp}^{1}(\mathrm{IC} 95 \%)^{2}$ & & & & \multicolumn{3}{|c|}{ - } & \multicolumn{3}{|c|}{ - } \\
\hline p-valor ${ }^{3}$ & \multicolumn{3}{|c|}{1,0000} & \multicolumn{3}{|c|}{0,5507} & \multicolumn{3}{|c|}{1,0000} \\
\hline
\end{tabular}


(Conclusão)

\begin{tabular}{|c|c|c|c|c|c|c|c|c|c|}
\hline \multirow{2}{*}{ Variáveis } & \multicolumn{3}{|c|}{18 a 25 anos $(n=31)$} & \multicolumn{3}{|c|}{26 a $44 \operatorname{anos}(n=132)$} & \multicolumn{3}{|c|}{45 anos ou mais $(n=72)$} \\
\hline & $\mathbf{N}$ & HPV & $\%$ & $\mathbf{n}$ & HPV & $\%$ & $\mathbf{n}$ & HPV & $\%$ \\
\hline \multicolumn{10}{|l|}{ Tabagismo } \\
\hline Sim & 2 & 1 & $50 \%$ & 14 & 5 & $35 \%$ & 7 & 4 & $57 \%$ \\
\hline Não & 29 & 11 & $37 \%$ & 118 & 40 & $33 \%$ & 65 & 25 & $38 \%$ \\
\hline $\mathrm{ORp}^{1}(\mathrm{IC} 95 \%)^{2}$ & \multicolumn{3}{|c|}{$1,63(0,09-28,90)$} & \multicolumn{3}{|c|}{$1,0(0,34-3,44)$} & \multicolumn{3}{|c|}{$2,1(0,44-10,33)$} \\
\hline $\mathrm{p}$-valor ${ }^{3}$ & \multicolumn{3}{|c|}{1,0000} & \multicolumn{3}{|c|}{0,9996} & \multicolumn{3}{|c|}{0,4286} \\
\hline \multicolumn{10}{|l|}{ Etilismo } \\
\hline Sim & 13 & 5 & $38 \%$ & 44 & 24 & $54 \%$ & 27 & 12 & $44 \%$ \\
\hline Não & 18 & 7 & $38 \%$ & 88 & 21 & $23 \%$ & 45 & 17 & $37 \%$ \\
\hline $\mathrm{ORp}^{1}(\mathrm{IC} 95 \%)^{2}$ & \multicolumn{3}{|c|}{$0,98(0,22-4,25)$} & \multicolumn{3}{|c|}{$3,8(1,77-8,26)$} & \multicolumn{3}{|c|}{$1,31(0,49-3,47)$} \\
\hline $\mathrm{p}$-valor ${ }^{3}$ & \multicolumn{3}{|c|}{1,0000} & \multicolumn{3}{|c|}{$<0,001^{*}$} & \multicolumn{3}{|c|}{0,6254} \\
\hline \multicolumn{10}{|l|}{ Número de gestações } \\
\hline Até 2 & 31 & 12 & $38 \%$ & 96 & 31 & $32 \%$ & 39 & 17 & $43 \%$ \\
\hline 3 ou mais & 0 & 0 & 0 & 36 & 14 & $38 \%$ & 33 & 12 & $36 \%$ \\
\hline $\mathrm{ORp}^{1}(\mathrm{IC} 95 \%)^{2}$ & \multicolumn{3}{|c|}{ - } & \multicolumn{3}{|c|}{$0,74(0,33-1,65)$} & \multicolumn{3}{|c|}{$0,63(0,24-1,67)$} \\
\hline $\mathrm{p}$-valor ${ }^{3}$ & \multicolumn{3}{|c|}{1,0000} & \multicolumn{3}{|c|}{0,5381} & \multicolumn{3}{|c|}{0,2462} \\
\hline \multicolumn{10}{|l|}{ Citologia cervical } \\
\hline Normal/Inflamatório & 22 & 4 & $18 \%$ & 98 & 20 & $20 \%$ & 47 & 15 & $31 \%$ \\
\hline Alterado & 9 & 8 & $88 \%$ & 34 & 25 & $73 \%$ & 25 & 14 & $56 \%$ \\
\hline $\mathrm{ORp}^{1}(\mathrm{IC} 95 \%)^{2}$ & \multicolumn{3}{|c|}{36,0 (infinito) } & \multicolumn{3}{|c|}{$10,8(4,37-26,82)$} & \multicolumn{3}{|c|}{$2,71(0,99-7,37)$} \\
\hline$p$-valor ${ }^{3}$ & \multicolumn{3}{|c|}{0,1677} & \multicolumn{3}{|c|}{$<0,001 *$} & \multicolumn{3}{|c|}{0,0763} \\
\hline
\end{tabular}

(-) Dados numéricos igual a zero.

$1 \mathrm{ORp}=$ Razão de Chances de Prevalência.

2 IC95\% = Intervalo de confiança a 95\%.

3 Teste do qui-quadrado ou teste exato de Fisher (nivel de significancia $\leq 0,05$ ).

*Associacao estatisticamente significante $(\mathrm{p} \leq 0,05)$. 